\title{
Construction and Characterization of a Novel Recombinant Attenuated and Replication-Deficient Candidate Human Adenovirus Type 3 Vaccine: "Adenovirus Vaccine Within an Adenovirus Vector"
}

\author{
Yuqian Yan ${ }^{1}$ Shuping Jing ${ }^{1,2} \cdot$ Liqiang Feng $^{3} \cdot$ Jing Zhang ${ }^{4} \cdot$ Zhiwei Zeng $^{1} \cdot$ Min $\mathrm{Li}^{1} \cdot$ Shan Zhao ${ }^{1} \cdot$ \\ Junxian $\mathrm{Ou}^{1} \cdot$ Wendong $\operatorname{Lan}^{1} \cdot$ Wenyi Guan ${ }^{1} \cdot$ Xiaowei $\mathrm{Wu}^{1} \cdot$ Jianguo $\mathrm{Wu}^{4} \cdot$ Donald Seto ${ }^{5} \cdot$ Qiwei Zhang $^{1,4}$ (i)
}

Received: 22 February 2020 / Accepted: 13 April 2020/ Published online: 26 May 2020

(c) Wuhan Institute of Virology, CAS 2020

\begin{abstract}
Human adenoviruses (HAdVs) are highly contagious and result in large number of acute respiratory disease (ARD) cases with severe morbidity and mortality. Human adenovirus type $3(\mathrm{HAdV}-3)$ is the most common type that causes ARD outbreaks in Asia, Europe, and the Americas. However, there is currently no vaccine approved for its general use. The hexon protein contains the main neutralizing epitopes, provoking strong and lasting immunogenicity. In this study, a novel recombinant and attenuated adenovirus vaccine candidate against HAdV-3 was constructed based on a commercially-available replication-defective HAdV-5 gene therapy and vaccine vector. The entire HAdV-3 hexon gene was integrated into the E1 region of the vector by homologous recombination using a bacterial system. The resultant recombinants expressing the HAdV-3 hexon protein were rescued in AD293 cells, identified and characterized by RT-PCR, Western blots, indirect immunofluorescence, and electron microscopy. This potential vaccine candidate had a similar replicative efficacy as the wild-type HAdV-3 strain. However, and importantly, the vaccine strain had been rendered replication-defective and was incapable of replication in A549 cells after more than twentygeneration passages in AD293 cells. This represents a significant safety feature. The mice immunized both intranasally and intramuscularly by this vaccine candidate raised significant neutralizing antibodies against HAdV-3. Therefore, this recombinant, attenuated, and safe adenovirus vaccine is a promising HAdV-3 vaccine candidate. The strategy of using a clinically approved and replication-defective HAdV-5 vector provides a novel approach to develop universal adenovirus vaccine candidates against all the other types of adenoviruses causing ARDs and perhaps other adenovirus-associated diseases.
\end{abstract}

Keywords Adenovirus vaccine - Human adenovirus type 3 (HAdV-3) - Replication-deficient adenovirus vector . Immunity in BALB/c mice $\cdot$ Recombination

\section{Introduction}

Human adenovirus (HAdV) is a wide spectrum virus that causes multiple systemic infections, such as acute respiratory disease (ARD), epidemic keratoconjunctivitis, hemorrhagic cystitis, gastroenteritis, as well as other

Yuqian Yan and Shuping Jing have contributed equally to this work.

Qiwei Zhang

zhangqw@smu.edu.cn

1 Guangdong Provincial Key Laboratory of Tropical Disease Research, School of Public Health, Southern Medical University, Guangzhou 510515, China

2 Microbiological Laboratory, Zhuhai Center for Disease Control and Prevention, Zhuhai 519000, China diseases (Lion 2014; Zhao et al. 2014). Using the whole genome sequence as a means of typing, 103 genotypes are currently recognized and classified into seven species A-G (Seto et al. 2011; Zhang et al. 2017). Among the genotypes of HAdV causing ARD, type 3 (HAdV-3) is the most common one reported to WHO (Echavarría 2009); it is particularly prevalent among children. Outbreaks of

3 Guangzhou Institutes of Biomedicine and Health, Chinese Academy of Sciences, Guangzhou 510530, China

4 Guangdong Provincial Key Laboratory of Virology, Institute of Medical Microbiology, Jinan University, Guangzhou 510632, China

5 Bioinformatics and Computational Biology Program, School of Systems Biology, George Mason University, Manassas, VA 20110, USA 
HAdV-3 infections are reported globally and recently, including the United States (Lebeck et al. 2009), Brazil (Pereira et al. 2016), the United Kingdom (Alkhalaf et al. 2015), Korea (Lee et al. 2010), Australia (Harley et al. 2001), Canada (Yeung et al. 2009), Singapore (Coleman et al. 2019), Malaysia ( $\mathrm{Li}$ et al. 2018), Japan (Fujimoto et al. 2008), and China (Zhang et al. 2006; Tsou et al. 2012; Wo et al. 2014; Chen et al. 2016; Yu et al. 2016; Lu et al. 2017; Lin et al. 2019; Zhao et al. 2019). Despite these widespread and recurring outbreaks, there is no specific drug or vaccine approved for use against HAdV-3 infections.

The protein capsid of adenovirus comprises 240 hexon trimers and 12 penton bases and fibers, of which the hexon protein contains the major neutralizing epitope ("epsilon") (Crawford-Miksza and Schnurr 1996; Singh et al. 2015). Each hexon monomer is encoded by approximately 900 amino acid residues that form a base (Rux et al. 2003), which is relatively sequence-conserved, and three tower domains that occur as loops lying on the exterior surface of the virion (Rux and Burnett 2000). These loops contain several hypervariable regions, noted as HVR1-HVR6 in loop 1 and HVR7 in loop 2 (Cheng et al. 2018), that are noted collectively as the epsilon epitope. This epsilon epitope provokes the host immune system into producing adenovirus serotype-specific antibodies that are highly effective and long-lasting (Rux et al. 2003; Russell et al. 2006; Pichla-Gollon et al. 2007; Radin et al. 2014; Tian et al. 2015).

Paradoxically, HAdV vectors have been widely and safely used in gene therapy protocols (Stecher et al. 2003; Holterman et al. 2004; Stone et al. 2005; Toth and Wold 2010; Wold and Toth 2013; Duffy et al. 2017). Several drugs incorporating adenoviruses as delivery vectors have been clinically approved and used in patients successfully (Liu and Kirn 2008; Räty et al. 2008). Additionally, HAdVs are the vector foundations of vaccines developed against many pathogens, including Ebola virus (Milligan et al. 2016; Shukarev et al. 2017), HIV (Schooley et al. 2010; Baden et al. 2014; Gu et al. 2014), avian influenza virus (Peters et al. 2013; Scallan et al. 2013), and foot-andmouth disease virus (Pena et al. 2008), as well as highly contagious bacteria associated with diseases, e.g., tuberculosis (Smaill et al. 2013).

In this study, the commercially-available and gene therapy use approved replication-defective HAdV-5 vector was used to construct a recombinant attenuated human adenovirus type 3 vaccine (Ginn et al. 2018). The complete hexon gene of HAdV-3 GZ01 was cloned into the AdEasy ${ }^{\mathrm{TM}}$ Adenoviral Vector, and this type-specific antigen was expressed when the recombinant adenovirus vaccine was inoculated into mice. This complete hexon protein with native conformation in theory was expressed successfully and neutralizing antibodies were produced. The recombinant vaccine is expected to be used in the prevention of ARD outbreaks caused by HAdV-3 infections, and to serve as a model using adenovirus vectors for the construction of other vaccines against additional important serotypes of adenoviral respiratory pathogens.

\section{Materials and Methods}

\section{Cells, Virus Strains and Viral Genome}

Replication-deficient adenoviruses were cultured in AD293 cells (purchased from Stratagene; CA, USA), which were grown in Dulbecco's modified Eagle's medium (DMEM) supplemented with $100 \mathrm{IU}$ penicillin/mL, $100 \mu \mathrm{g}$ streptomycin $/ \mathrm{mL}$, and $10 \%(\mathrm{v} / \mathrm{v})$ fetal calf serum. The HAdV-3 GZ01 strain (accession number DQ099432) was originally isolated from a child with ARD (Zhang et al. 2006) and cultured in A549 cells. Viral genomic DNA was extracted by our modified protease $\mathrm{K}$ digestion method.

\section{Bacteria, Plasmids and Enzymes}

E. coli strains DH5 $\alpha$ and BJ5183 are laboratory stocks. Plasmids pShuttle and pAdEasy were purchased from AdEasy ${ }^{\mathrm{TM}}$ Adenoviral Vector System (Stratagene; CA, USA). Platinum ${ }^{\circledR}$ Taq DNA polymerase with high-fidelity and restriction enzymes were purchased from Life Technologies Corporation (Carlsbad, CA, USA) and New England Biolabs (MA, USA), respectively.

\section{Construction of rAd3H Recombinant Adenovirus Containing the Hexon Gene of HAdV-3}

In brief, the complete hexon gene of HAdV-3 GZ01 was cloned into pShuttle and recovered as recombinant psAd3H. This pShuttle vector was then subjected to homologous recombination with pAdEasy, using the highly efficient homologous recombination system in E. coli BJ5183 according to the manufacturer's protocol. Finally, the $\mathrm{rAd} 3 \mathrm{H}$ recombinant virus was rescued by transfection with prAd3H in AD293 cells and cultured for 20 generations. This construction strategy is presented as Fig. 1, with the detailed steps as follows.

First, Platinum ${ }^{\circledR}$ taq DNA polymerase with high-fidelity was used to PCR amplify the complete hexon gene of HAdV3 GZ01, using the primer pairs Ad3-GZ01-EcoR V-HexF (5'-TGAGATATCGCCACCATGGCCACCCCATC- $\left.{ }^{\prime}\right)$ and Ad3-GZ01-HexR-Xho I (5'-AGACTCGAGTTATGTGG TGGCGTTGCC-3'). Following, two restriction sites, EcoR V and Xho I, were added onto opposite ends of this amplified hexon gene and the PCR products were purified by PCR Clean up Kit (Axygen; New York, USA). Both the PCR products 


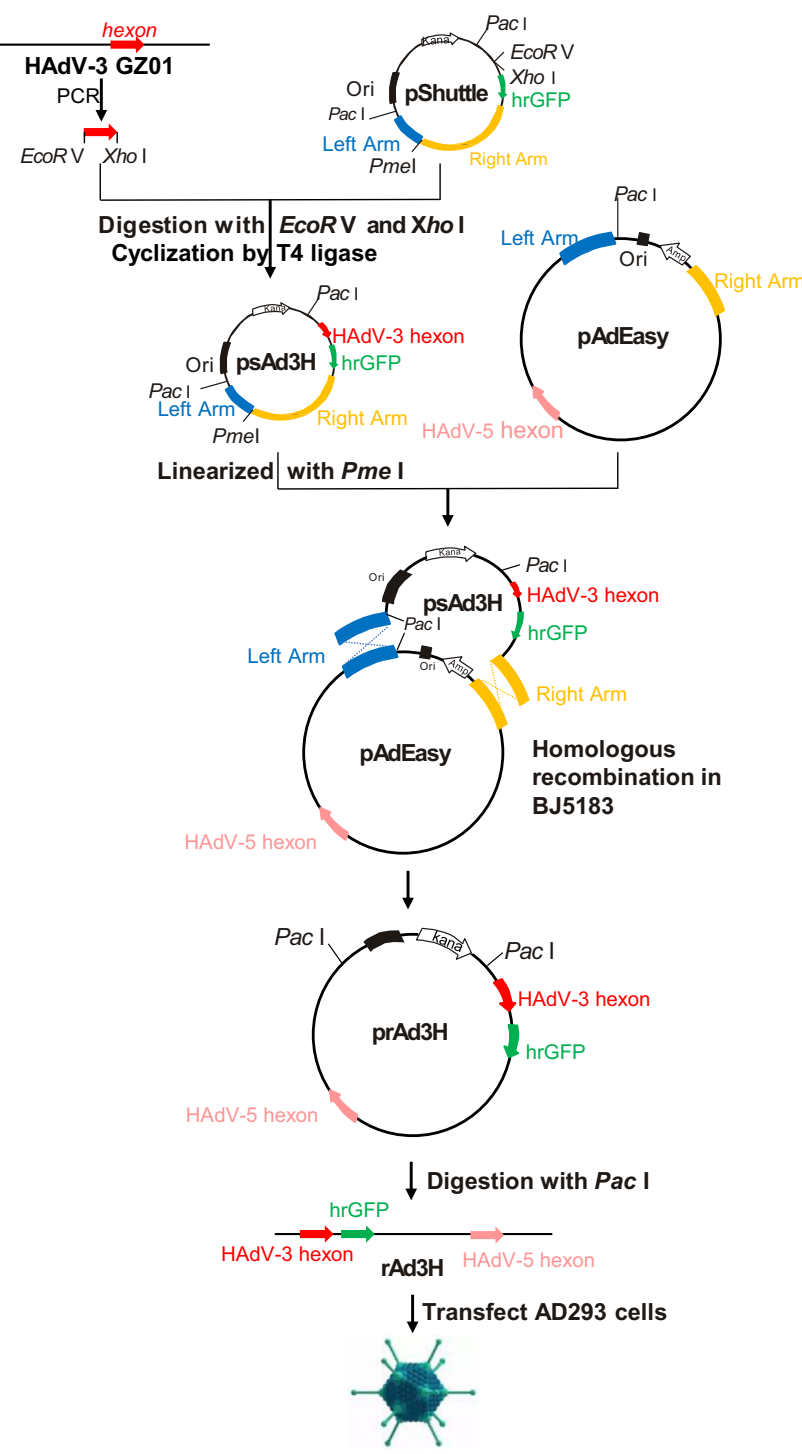

Fig. 1 Construction of recombinant adenovirus vector $\operatorname{prAd} 3 \mathrm{H}$ and the rescue of recombinant adenovirus $\mathrm{rAd} 3 \mathrm{H}$. The complete hexon gene from HAdV-3 was PCR-amplified and cloned into pShuttle vector. The resultant plasmid psAd3H and pAdEasy were ligated by means of homologous recombination and produced $\operatorname{prAd} 3 \mathrm{H}$, which was further linearized and transfected into AD293 cells. After continuous culture and passages, the recombinant vaccine strain rAd3H was rescued successfully.

and the pShuttle plasmid were digested by EcoR V and Xho I in $37^{\circ} \mathrm{C}$, respectively, permitted to reanneal, and then ligated using T4 DNA Ligase at $16^{\circ} \mathrm{C}$ for $8 \mathrm{~h}$. After ligation, the recombinant was transformed into $200 \mu \mathrm{L}$ E. coli DH5 $\alpha$ competent cells. In the next $12-16 \mathrm{~h}$, the cells were grown in LB agar plates, selected by ampicillin. After selection and amplification of each single clone, the plasmid was extracted and verified by PCR and sequencing, and archived as psAd3H.

Second, approximately $100 \mathrm{ng}$ of pAdEasy and $700 \mathrm{ng}$ of psAd3H linearized with Pme I both were transformed into E. coli BJ5183 competent cells which contained recombinase, and then selected on LB-Kanamycin agar plates. As the small colonies were potential candidate recombination clones (Zhang et al. 2009), they were selected and further identified by PCR screening, using HAdV-3 type-specific primers (Ad3F 5'-AAGACATTACCACTACTGAAGGAGAAGAA- $3^{\prime}$ and Ad3R $5^{\prime}$-CGCTAAAGCTCCTGCAACAGCAT-3') (Han et al. 2013) and producing a PCR product of approximately 300 bp. This PCR-positive plasmid DNA was then amplified in DH5 $\alpha$ cells, and additionally identified by sequencing and restriction enzyme digestion $(E c o \mathrm{R}$ I, EcoR V, Not I, Sal I, and Xho I). Plasmid Miniprep Kit (Axygen; New York, USA) was used to purify this recombinant plasmid prAd3H.

Third, the prAd3H plasmid was digested with $P a c$ I to release the linear adenovirus genomic DNA, which was then transfected into AD293 cells using Lipofectamine LTX (Life Technologies Corporation; Carlsbad, CA, USA). The cells were examined daily for 10 days for evidence of increased fluorescence and cytopathic effect, which signaled successful transfection and packaging. After 10 days, the cultures were frozen and thawed thrice and then centrifuged at $12,000 \times g$ for $10 \mathrm{~min}$. The supernatant was used to infect the AD293 cells. Cells were cultured for about 7 days. Fluorescence and cytopathic effect were monitored each day. The recombinant adenovirus rAd3H was rescued successfully when both GFP and CPE were observed.

\section{Reverse Transcription Polymerase Chain Reaction (RT-PCR) and Western Blot Analysis}

The transcription of HAdV-3 hexon gene of rAd3H was confirmed by RT-PCR analysis as follows: Total RNA was extracted by RNAiso (TAKARA; Tokyo, Japan) and reverse transcribed into cDNA using the primer HVRR (5'TTTCTGAAGTTCCACTCGTAGGTGTA-3'). This resultant cDNA was used as the template in a PCR amplification with the primer pairs, HVRF1 (5'-CAGGATGCTTCGGAGTACCTGAG-3') and HVRR (Zhang et al. 2020). The PCR product was identified using agarose gel electrophoresis and DL10000 markers. Subsequent Western blot analysis confirmed expression of the hexon protein. A culture of $\mathrm{rAd} 3 \mathrm{H}$ was frozen and thawed three times, centrifuged at $1000 \times g$ for $10 \mathrm{~min}$, and the supernatant was collected. The samples were added to loading buffer, boiled for $10 \mathrm{~min}$, electrophoresed in 5\% concentrated gel, and $10 \%$ Tris-Tricine SDS-PAGE. After PAGE, the proteins were transferred onto nitrocellulose membrane by electrophoresis at $25 \mathrm{~V}$ for $40 \mathrm{~min}$. The membrane was probed first by binding with mouse anti-HAdV-3-hexon antibody (Guangzhou HuYanSuo Medical Technology; Guangzhou, China) as the epitope-specific antibody 
(1:1000 dilution), and followed with goat anti-mouse IgG-HRP (Bioworld; Georgia, USA) as the secondary amplifying antibody. Luminol and hydrogen peroxide were used as chromogenic substrates to score the fluorescence signal.

\section{Indirect Immunofluorescence Assay}

Indirect immunofluorescence assay was performed to detect the virus titer of HAdV-3. $2 \times 10^{4}$ AD293 cells were seeded into each well of a 96-well plate. After an 18-24-h culture, the cells were infected with $100 \mu \mathrm{L}$ $\mathrm{HAdV}-3$. At $48 \mathrm{~h}$ post infection, the cells were fixed in methanol, precooled in $-20{ }^{\circ} \mathrm{C}$, and incubated at $37{ }^{\circ} \mathrm{C}$ for 30 min with $1 \%$ BSA. The mouse anti-HAdV-3-hexon monoclonal antibody, at a dilution of 1:1000 in PBST, was added to wells and incubated at $37{ }^{\circ} \mathrm{C}$ for an hour. FITCconjugated goat anti-mouse $\operatorname{IgG}(1: 10000)$ was then added and the mixture incubated at $37{ }^{\circ} \mathrm{C}$ for an hour. The number of green fluorescence cells were counted under reversed fluorescence microscopy and virus titer were calculated by fluorescence forming unit (FFU) with the formula of $\mathrm{FFU} / \mathrm{mL}=10^{\mathrm{n}} \times$ average number of GFP positive cells/well $\times 10(\mathrm{n}$ : dilution times $)$.

\section{Transmission Electron Microscopy (TEM) Observation}

AD293 cells infected with rAd3H viruses were grown and harvested when $90 \%$ CPE was observed. These cells were frozen and thawed three times, centrifuged at $12,000 \times g$ for $10 \mathrm{~min}$. Supernatant containing viral particles was collected, upon which a grid covered with carbon support formvar film was floated, for $2 \mathrm{~min}$. This was stained with $10 \mu \mathrm{L}$ sodium phosphor-tungstate, dried at $40{ }^{\circ} \mathrm{C}$ for $10 \mathrm{~min}$, and the vaccine strained $\operatorname{rAd} 3 \mathrm{H}$ was observed using a TEM technique (FEI Tecnai ${ }^{\mathrm{TM}}$ electron microscope).

\section{Adenovirus Growth Characteristics and Stability Assay}

AD293 cells were seeded into 6-well plates with $5 \times 10^{5}$ cells per well. After 16-24 h, the cells were infected with $5 \times 10^{4}$ DNA copies of rAd3H, rAd5 or HAdV-3 strain GZ01, respectively. Cultures were harvested after 12, 24, $36,48,72,84,96,108$, or $120 \mathrm{~h}$ post-infection. These cultures were frozen and thawed three times, then the supernatant was collected after centrifugation. The viral DNA copies at all the time-points were quantified by realtime PCR using the primers pentonF $\left(5^{\prime}\right.$-ACCACCGTCAGTGAAAACG- $3^{\prime}$ ) and pentonR (5'-TATGCCCAGKGCCTTGT- $3^{\prime}$ ) to quantify the genomic DNA copy number. Using HAdV-5 DNA as the template and pentonF and pentonR as primers, the PCR product was ligated into the pMD18-T vector (TAKARA; Tokyo, Japan). The resultant plasmid was used as the standard for real-time PCR quantification. The viral stability of the recombinant vaccine strain $\mathrm{rAd} 3 \mathrm{H}$ after continuous passages was further tested. Briefly, the virus was cultured in AD293 cells and passaged for more than 20 generations. At every five generations, adenovirus genomic DNA was extracted; these served as templates for the PCR amplification of HAdV-3 hexon gene, which was then further characterized by DNA sequencing. The 20th generation of rAd3H harvested from the AD293 cells culture was further inoculated into A549 cells and re-cultured for 7 days to determine if there were replication-competent mutants resulting from the continuous passages of $\mathrm{rAd} 3 \mathrm{H}$ in $\mathrm{AD} 293$ cells. If no green fluorescence was observed during the culturing in A549 cells, it meant that no replication-competent mutants occurred. Therefore, the stability of the recombinant vaccine strain was verified.

\section{Virus Purification and Concentration}

Discontinuous density-gradient centrifugation was used to purify and concentrate the recombinant adenovirus $\mathrm{rAd} 3 \mathrm{H}$ as follows: $6 \mathrm{~mL} 1.2 \mathrm{~g} / \mathrm{mL} \mathrm{CsCl}$ was first added into the centrifuge tube with $8 \mathrm{~mL} 1.4 \mathrm{~g} / \mathrm{mL} \mathrm{CsCl}$ slowly added to the bottom subsequently, resulting in a stratification. On the top of the $\mathrm{CsCl}, 20 \mathrm{~mL}$ of the virus culture was then carefully layered, and the tube was centrifuged at $141,000 \times g$ at $4{ }^{\circ} \mathrm{C}$ for $3 \mathrm{~h}$. The virus band was then extracted and transferred to a dialysis bag (MWCO: $14,000 \mathrm{Da}$ ) and dialyzed in a buffer at $4{ }^{\circ} \mathrm{C}$, with two buffer changes, to remove the $\mathrm{CsCl}(10 \mathrm{mmol} / \mathrm{L}$ Tris $-\mathrm{Cl}$ $\mathrm{pH}$ 8.0, $2 \mathrm{mmol} / \mathrm{L} \mathrm{MgCl}_{2}$, and 5\% sucrose). Purified virus was titrated by a modified fluorescence-forming unit assay (Philipson 1961). Briefly, AD293 cells were infected with ten-fold diluted $\mathrm{rAd} 3 \mathrm{H}$ virus in 96 well-plates. After $48 \mathrm{~h}$ of incubation, $\mathrm{rAd} 3 \mathrm{H}$ infected cells are directly observed under fluorescent microscopy. Wells with suitable virus dilution were selected for counting the number of fluorescent cells. The virus titer of FFU was calculated as described above.

\section{Animal Immunization}

Four to six-week-old female specific-pathogen-free BALB/c mice were purchased from the Laboratory Animal Center of Southern Medical University (Guangzhou, China) and kept in individual ventilated cage system. Six mice in each group were either inoculated with $1.8 \times 10^{8} \mathrm{FFU} / \mathrm{kg}$ of HAdV-3 GZ01 or immunized with the $\mathrm{rAd} 3 \mathrm{H}$ recombinant vaccine by the intranasal route or intramuscular route, respectively. 
The negative control group was inoculated with PBS of the same volume. The mice were boosted with the same virus or vaccine strain at day 14 post inoculation. At days 21, 28, 35 and 42 , sera from three mice in each group were collected and the $50 \%$ neutralizing antibody titer was determined by microculture neutralization test.

\section{Microculture Neutralization Test}

A microculture neutralization test was performed to determine the HAdV-3 neutralizing antibody titers using a previously described protocol (Zhang et al. 2009). Sera from mice immunized with $\mathrm{rAd} 3 \mathrm{H}$ by either intramuscular injection or intranasal inoculation were tested for the presence of HAdV-3 neutralizing antibody. The 50\% virus neutralization titers were calculated by Reed-Muench method.

\section{Results}

\section{Construction and Screening of Recombinant prAd3H Plasmid Inserted by the Hexon Gene of HAdV-3}

A pShuttle plasmid psAd3H containing the complete hexon gene, which contains the epsilon epitope of HAdV-3, was constructed. This construct also contained the left and right homologous regions that enable homologous recombination with pAdEasy to produce infectious clone prAd3H (Fig. 1). The resultant plasmid was confirmed by DNA sequencing, which not only provided confirmation of the complete hexon gene sequence but also verified that no mutations were introduced. Prior to sequencing, as a rapid check, restriction endonuclease analysis (REA) was performed to characterize the recombinant plasmid rapidly. Restriction maps generated with five restriction endonucleases were consistent with the in silico restriction maps predicted by the Vector NTI 11.5.1 software (Invitrogen Corp; San Diego, CA, USA) (Zhao et al. 2014; Yu et al. 2016; Zhang et al. 2017; Pan et al. 2018) (Fig. 2).

\section{Rescue of Recombinant Adenovirus rAd3H Expressing HAdV-3 Hexon Gene}

Recombinant plasmid prAd3H was linearized by Pac I and transfected into AD293 cells to rescue the recombinant adenovirus rAd3H. Green fluorescence was observed under fluorescence microscopy and the fluorescence density was noted to increase each subsequent day, an indication that the recombinant adenovirus was replicating. At day 10 post-transfection, the virus culture was frozen and thawed three times; after centrifugation, the supernatant was
A

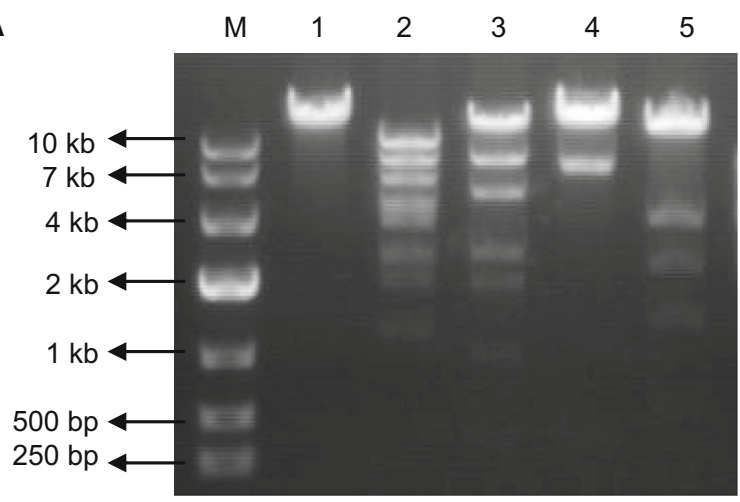

B

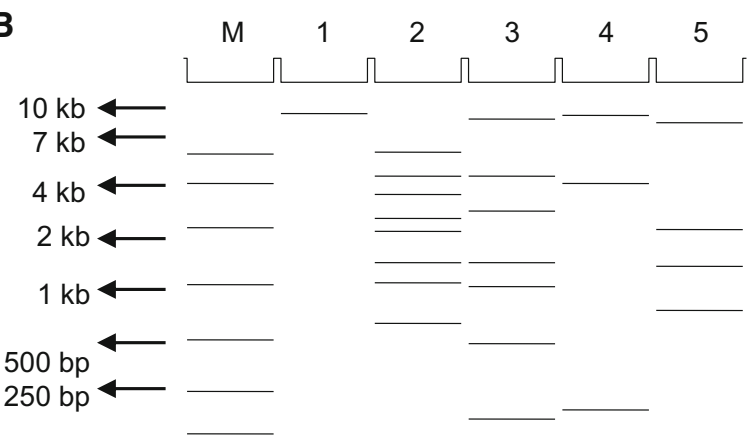

Fig. 2 Restriction enzyme analysis of prAd3H. A Lanes: (M) DL10000 marker; (1) $\operatorname{prAd} 3 \mathrm{H} / E c o \mathrm{R} \mathrm{I}$; (2) $\operatorname{prAd} 3 \mathrm{H} / E c o \mathrm{R} \mathrm{V}$; (3) $\mathrm{prAd} 3 \mathrm{H} /$ Not I; (4) prAd3H/Sal I; and (5) prAd3H/Xho I. B in silico maps predicted by the Vector NTI 11.5.1 software (Invitrogen Corp; San Diego, CA, USA).

inoculated into AD293 cells. Green fluorescence and CPE were observed and recorded at day 8 post-infection (Fig. 3). The increased fluorescence and CPE suggested

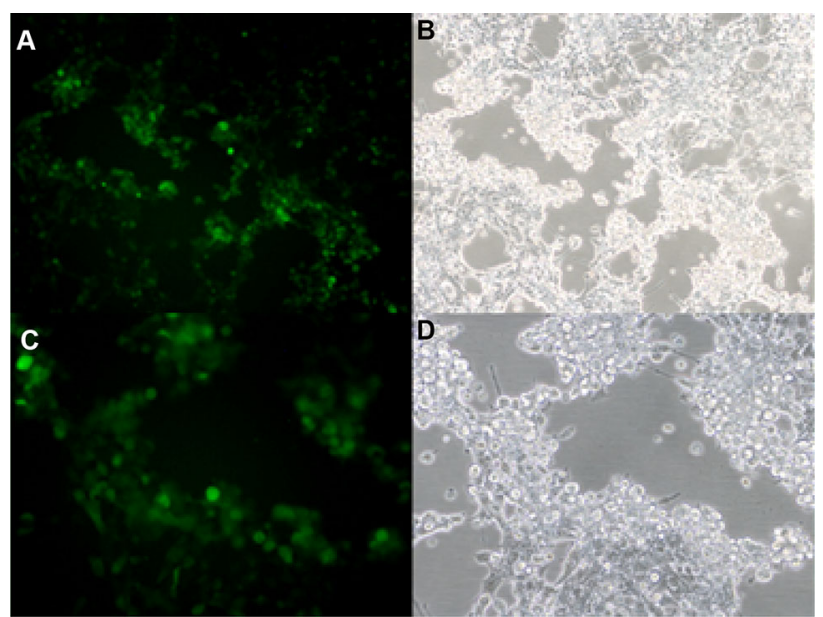

Fig. 3 Fluorescence and CPE of AD293 cells were observed at day 8 post-infection by $\mathrm{rAd} 3 \mathrm{H}$ recombinant adenoviruses. The AD293 cells infected with the $\mathrm{rAd} 3 \mathrm{H}$ recombinant virus were observed. A, C Green fluorescence. B, D Normal vision. A, B $100 \times$; C, D 200×. 
that the recombinant virus $\mathrm{rAd} 3 \mathrm{H}$ was rescued successfully from AD293 cells.

\section{RT-PCR and Western Blot Assay to Identify the Transcription and Expression of HAdV-3 Hexon Protein}

The transcription of the rAd3H hexon gene was confirmed by RT-PCR. A rAd5 virus that containing adenovirus type 5 genome except for E1 and E3 regions was used as a positive control. Viral RNA was extracted from a rAd3H culture and digested with DNase. After digestion, the presumed viral RNA was used as a PCR template for amplifying the hexon gene as a check for any residual DNA. No PCR products were found from these samples, which indicated that the extracted RNA did not contain viral genomic DNA (Fig. 4A). After reverse transcription, the cDNA was used as a PCR template to validate the insert. The resultant amplification of the hexon gene confirmed the $\mathrm{rAd} 3 \mathrm{H}$ recombinant adenoviruses. (Fig. 4B).

Expression of the HAdV-3 hexon protein in the culture supernatant was also detected by Western blots (Fig. 4C), in which HAdV-3 specific mouse monoclonal antibody was used to identify HAdV-3 hexon. The rAd5 adenovirus was used as the negative control, as it contained the HAdV-5 hexon gene but not the HAdV-3 hexon gene. The HAdV-3 hexon protein of $108 \mathrm{kDa}$ was confirmed in the $\mathrm{rAd} 3 \mathrm{H}$ recombinant vaccine strain while no band was found in the rAd5 and mock samples, indicating that during the culture, the HAdV-3 hexon protein of $\operatorname{rAd} 3 \mathrm{H}$ can be expressed successfully.

\section{Electron Microscopic Observation of the Vaccine Strain rAd3H}

The harvested viral culture from $\mathrm{rAd} 3 \mathrm{H}$ was negativestained by sodium phosphor-tungstate and observed under electron microscopy. Typical adenovirus particles, $70-90 \mathrm{~nm}$ in diameter, were clearly visible (Fig. 5). This further confirmed the successful infection and replication of the recombinant HAdV-3 vaccine in AD293 cells.

\section{Growth Characteristics of Recombinant Vaccine rAd3H}

The replication efficiencies of $\mathrm{rAd} 3 \mathrm{H}, \mathrm{rAd} 5$ and HAdV-3 strain GZ01 were compared by quantification of genomic DNA copies using a real-time PCR method. Primers targeting the conserved region of the penton base gene to represent the genomic DNA number were used in the realtime PCR amplification. The two one-step growth curves of rAd3H and HAdV-3 strain GZ01 showed that the replication efficacy of $\mathrm{rAd} 3 \mathrm{H}$ viruses were similar to that of

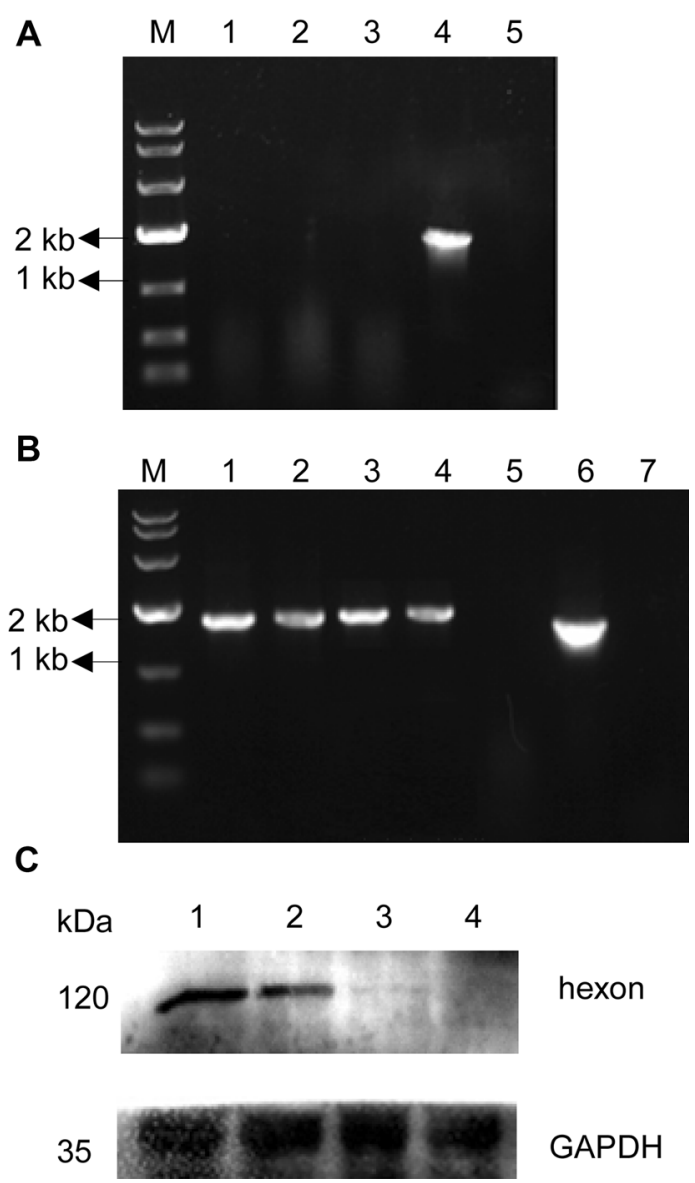

Fig. 4 RT-PCR analysis of rAd3H hexon transcription $(\mathbf{A}, \mathbf{B})$ and Western blot confirmation of the HAdV-3 hexon protein expression from rAd3H (C). A RNA as template for PCR. Lanes: (M) DL10000 marker; (1) rAd3H; (2) rAd5; (3) mock; (4) PCR positive control; (5) PCR negative control; B cDNA as template for PCR. (M) DL10000 marker; (1-2) rAd3H; (3-4) rAd5; (5) mock; (6) PCR positive control; (7) PCR negative control; C Lanes: (1-2) rAd3H cell supernatant; (3) negative control, rAd5 cell supernatant; (4) mock, supernatant of AD293 cells.

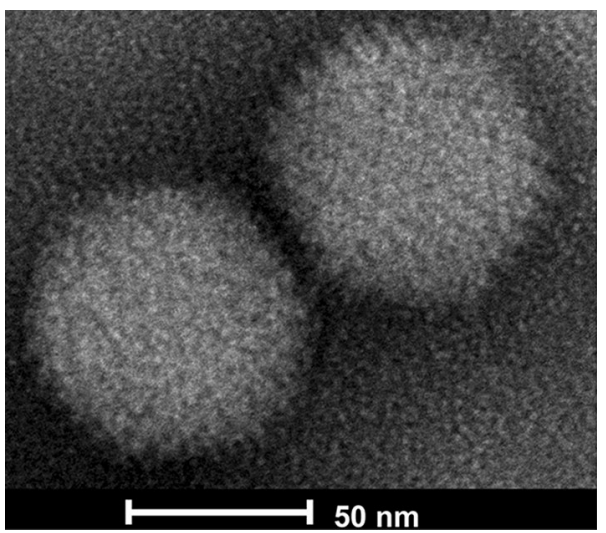

Fig. 5 Electron micrograph visualization of the $\operatorname{rAd} 3 \mathrm{H}$ recombinant vaccine virus. Negative staining of the particles recovered from a viral culture shows adenovirus-like virions. Scale bar: $50 \mathrm{~nm}$. 


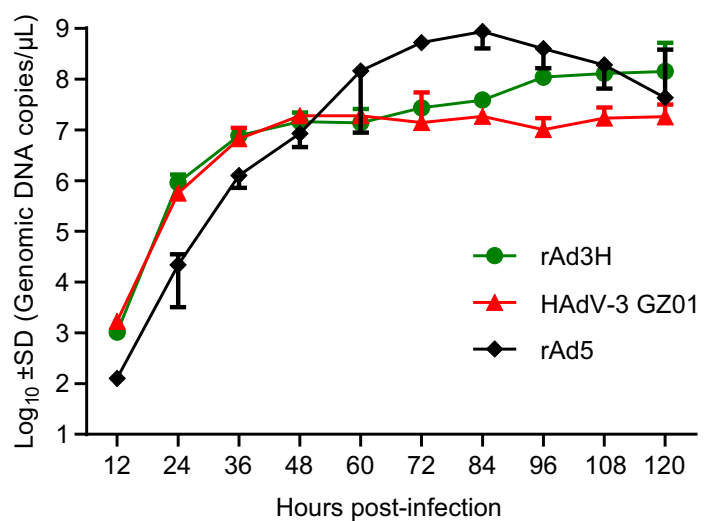

Fig. 6 Replication dynamics curves of $\mathrm{rAd} 3 \mathrm{H}$ bearing HAdV-3 hexon gene, rAd5 and HAdV-3 wild-type strain GZ01. AD293 cells were infected by the two viruses and harvested at 12, 24, 36, 48, 60, 72, 84, 96,108 , and $120 \mathrm{~h}$ post infection. Viral genomic DNA copy numbers in cells and supernatants were determined by real-time PCR for the penton base region.

HAdV-3 wild-type strain in AD293 cells, both of which increased similarly during the $60 \mathrm{~h}$ post infection. The peaks of DNA copy number of both rAd5 and rAd3H strains were higher than HAdV-3 GZ01 strain, which might be because HAdV-5 genomic DNA is the backbone of both strains and the viruses are cultured in AD293 cells which highly expressed HAdV-5 E1A and E1B genes that promote the HAdV-5 DNA replication (Fig. 6).

\section{Stability of Recombinant Vaccine Strain rAd3H}

To assess the stability of the insert, recombinant vaccine strain $\mathrm{rAd} 3 \mathrm{H}$ was passaged in AD293 cells for at least 20 generations. The virus stability of $\mathrm{rAd} 3 \mathrm{H}$ harvested from the 20 generations of culture was verified by PCR amplification and DNA sequencing of the HAdV-3 and HAdV-5 hexon genes. There were no amino acid mutations detected in the HAdV-3 and HAdV-5 hexon gene of rAd3H upon sequence verification. This indicated that both hexon genes in this recombinant vaccine strain are well compatible with each other during the viral replication. As expected, both fluorescence and CPE were observed in the AD293 cells infected by $\mathrm{rAd} 3 \mathrm{H}$ from the first to the twentieth generations (Fig. 7A-7D). However, no green fluorescence was detected in the A549 cells infected by $\mathrm{rAd} 3 \mathrm{H}$ which was the culture from the first to the twentieth generations of rAd3H in A549 cells (Fig. 7E-7H), which indicated no infectious viral particles produced in A549 cells. PCR amplification of the hexon gene from the virus culture in A549 cells showed no product, which indicated that rAd3H could only replicate in AD293 cells, but not in the A549 cells due to the E1 deletion. No reverse mutations occurred during the continuous passages of rAd3H in AD293 cells. All the results presented confirmed the stability of the vaccine stain rAd3H in AD293 cells (Fig. 7).

\section{Serum Antibody Titer in Immunized Mice}

Mice were either inoculated with HAdV-3 wild-type strain GZ01 or immunized with the $\mathrm{rAd} 3 \mathrm{H}$ recombinant vaccine by either the intranasal route or intramuscular route, respectively, to assess the antibody titer. At day 14 post inoculation/immunization, the mice were boosted with the same inoculant. Mouse sera were collected at day 21, 28, 35 , and 42 after the prime inoculation/immunization. The $50 \%$ neutralizing antibody titers of mouse sera in the intranasal or intramuscular groups collected at different

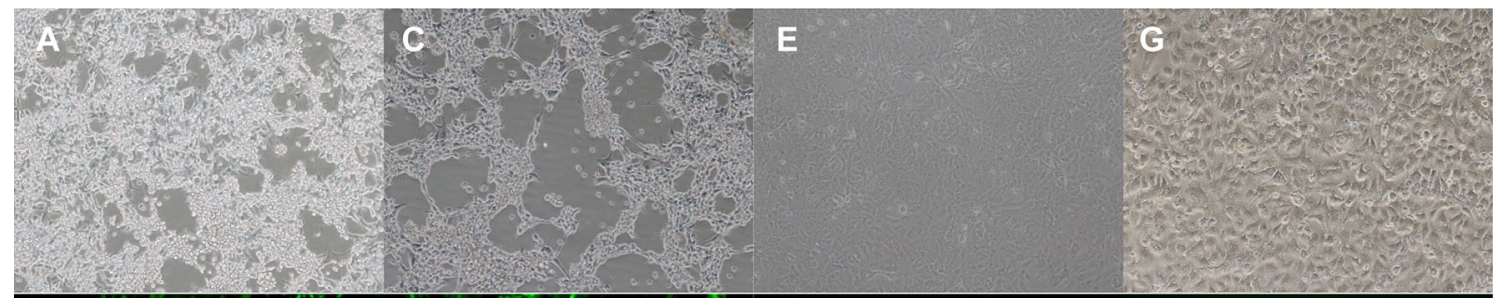

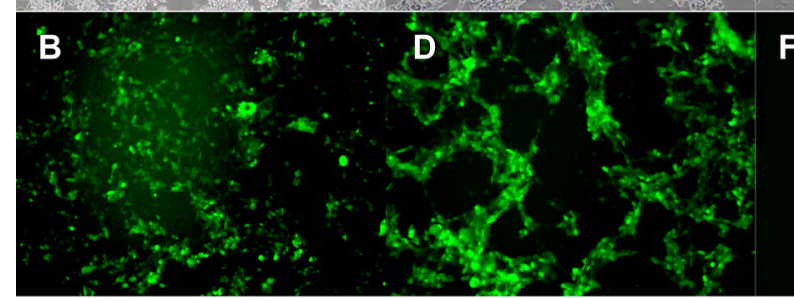

AD293, $1^{\text {st }}$ generation
AD293, $20^{\text {th }}$ generation

Fig. 7 Light and fluorescent microscopy observation of cells infected by the recombinant vaccine rAd3H. A, B AD293 cells infected by the 1st generation of $\mathrm{rAd} 3 \mathrm{H}$; C, D AD293 cells infected by the 20th generation of $\mathrm{rAd} 3 \mathrm{H} ; \mathbf{E}, \mathbf{F}$ A549 cells infected by the 1st generation

$\mathbf{F}$

A549, $1^{\text {st }}$ generation
H

of rAd3H cultured in A549 cells; G, H A549 cells infected by the 20th generation of rAd3H cultured in A549 cells. A, C, E, G are in fluorescence vision; $\mathbf{B}, \mathbf{D}, \mathbf{F}, \mathbf{H}$ are in white light vision $(100 \times)$ at day 5 post-infection. 
Table 1 Determination of the $50 \%$ neutralizing antibody titer in mouse sera by the microculture neutralization test.

\begin{tabular}{llllll}
\hline Serum collection time & \multicolumn{2}{l}{ rAd3H immunization } & & \multicolumn{2}{l}{ HAdV-3 wildtype GZ01 inoculation } \\
\cline { 2 - 3 } & Intranasal & Intramuscular & & Intranasal & Intramuscular \\
\hline D21 & $1: 250$ & $1: 270$ & & $1: 1000$ & $1: 1428$ \\
D28 & $1: 200$ & $1: 250$ & & $1: 1111$ & $1: 1000$ \\
D35 & $1: 167$ & $1: 333$ & & $1: 1111$ & $1: 1667$ \\
D42 & $1: 158$ & $1: 248$ & & $1: 916$ & $1: 1024$ \\
\hline
\end{tabular}

The mice were either immunized with the rAd3H recombinant vaccine or inoculated with HAdV-3 wildtype strain GZ01 intranasally or intramuscularly. At day 21, 28, 35, and 42 after the prime inoculation/ immunization, the neutralizing antibodies against HAdV-3 were titrated. time points were determined by the microculture neutralization test (Table 1). All of the mice immunized with rAd3H or HAdV-3 GZ01 virus intranasally or intramuscularly produced neutralizing antibodies. However, the mice immunized intramuscularly produced higher and more lasting antibody titers than the intranasally immunized mice. Therefore, apparently the intramuscular immunization was more effective than the intranasal immunization in provoking the immune response. In the wild-type GZ01-innoculated mice, higher neutralizing antibody titers were produced in both intranasal and intramuscular groups, which might be associated with the replication-competence of wild-type adenoviruses as compared with the replication-deficient $\mathrm{rAd} 3 \mathrm{H}$ vaccine strain.

\section{Discussion}

Human adenoviruses are highly contagious pathogens that are associated with several severe and fatal diseases including ARD (Jing et al. 2019; Zhang et al. 2019). ARD associated with HAdV-3 often results in severe morbidity and some fatalities. Additionally, among all of the adenovirus types causing ARD, HAdV-3 appears to be the most common found in pediatrics cases (Chang et al. 2008). There is no vaccine currently available for preventing HAdV-3 outbreaks. In fact, the only two vaccines available against HAdVs (types 4 and 7) are not available to the public. In this study, we provide a novel strategy for the construction of a candidate adenovirus type 3 vaccine that may serve as a template for developing similar vaccines for use against other pathogen HAdVs. The time-tested and safe HAdV-5 replication-deficient vaccine vector was used for the construction of this candidate vaccine strain that harbors the immunogenic epitope from human adenovirus type 3 hexon. We demonstrate that the administration of this recombinant and replication-deficient $\mathrm{rAd} 3 \mathrm{H}$ strain could elicit the significant increase of neutralizing antibodies against $\mathrm{HAdV}-3$; therefore, it could be considered a candidate vaccine strain to be used for the prevention of HAdV-3 infection or epidemics.

There are three methods used to construct an adenovirus vector: in vitro ligation, Cre/loxP system, homologous recombination in eukaryotic cells or in prokaryote cells method. The genome of human adenoviruses is about $35 \mathrm{~kb}$. Compared with the other methods, the method of homologous recombination in prokaryote cells has the advantages of a simpler protocol, higher recombination efficiency, and easier screening of positive clones. In this study, we used the homologous recombination in $E$. coli BJ5183 to construct a recombinant adenovirus vector to serve as the basis for a vaccine against a commonly circulating adenoviral respiratory pathogen. This recombinant DNA vector construction protocol consists of two vectors: an adenovirus backbone plasmid, pAdEasy, and a pShuttle plasmid. The pShuttle plasmid contains a multiple cloning site and a kanamycin resistance gene, which support the insertion and maintenance of a foreign gene, as well as provides for the rapid screening for recombination. A PCRderived product comprising the complete hexon gene (epsilon epitope) of adenovirus type 3 was directly cloned into the multiple cloning site of pShuttle using the EcoR V and Xho I restriction sites. The linearized psAd3H and pAdEasy were transformed into an engineered bacterial strain BJ5183 that was recA proficient and supplied the machinery necessary to execute the recombination event. Homologous recombination was carried out to obtain the vaccine vector bearing the complete HAdV-3 hexon gene. To overcome the undue homologous recombination during the bacterium culture, the recombinant time in BJ5183 was restricted to no more than $8 \mathrm{~h}$, then recombinant bacterial clones were screened by kanamycin resistance. Following the construction of the recombinant plasmid, the putative size of the insert allows for an initial screen, with additional identification, confirmation, and characterization by PCR, REA, DNA sequencing, and other assays.

The rAd3H vaccine contains most genes of HAdV-5 except the E1 and E3 regions, as well as the complete HAdV-3 hexon gene. The E1 region is essential for the 
assembly of infectious virus particles, so the replication of rAd3H can only take place in adenovirus packaging cell line like AD293 cells which express the E1 proteins of $\mathrm{HAdV}-5$, while $\mathrm{rAd} 3 \mathrm{H}$ cannot replicate in any kind of human cells. Therefore, the safety of $\mathrm{rAd} 3 \mathrm{H}$ recombinant adenovirus vaccine is guaranteed.

The recombinant vaccine $\mathrm{rAd} 3 \mathrm{H}$ expresses the complete HAdV-3 hexon gene in infected cells, which can maintain the native conformation of the whole HAdV-3 hexon protein. In theory, this will guarantee a better immune effect. However, mice immunized with recombinant vaccine $\operatorname{rAd} 3 \mathrm{H}$ produced lower neutralizing antibody titers than wild-type HAdV-3 strain. One of the causes may be that the capsid proteins of fiber and penton base in the wildtype viruses also provoke certain immunogenicity (GaherySegard et al. 1998; Feng et al. 2018). In rAd3H vaccine strain, no HAdV-3 fiber and penton protein were expressed, while there was in HAdV-3 wild-type strain. Another cause is the $\mathrm{rAd} 3 \mathrm{H}$ vaccine strain is replication deficient, which cannot replicate and express proteins well in normal cells. As the result, the induced humoral immunity by the wildtype strain in mice was stronger than replication deficient vaccine strain.

In summary, a recombinant and attenuated adenovirus vaccine candidate against HAdV-3 was constructed based on a commercially-available, replication-defective HAdV-5 vector that has been widely used in previous gene therapy protocols and vaccine development. This potential vaccine candidate had a similar replicative efficacy in AD293 cells as the wild-type HAdV-3 strain. The recombinant is also highly stable and could elicit significant neutralizing antibodies against HAdV-3, with both intranasal and intramuscular inoculation of mice. Therefore, this recombinant adenovirus vaccine is a promising vaccine candidate against HAdV-3. The strategy of using a clinically approved, previously used in gene therapy settings, and replication-defective HAdV-5 vector as the basis of the vaccine provides a novel approach for future development of additional adenovirus vaccine candidates against all of the other adenoviral genotypes causing ARDs and other diseases.

Acknowledgements This work was supported by Grants from the National Key Research and Development Program of China (2018YFE0204503), National Natural Science Foundation of China (31570155, 31370199), and Natural Science Foundation of Guangdong Province (2018B030312010), as well as the Guangzhou Healthcare Collaborative Innovation Major Project (201803040004, 201803040007).

Author Contributions QZ designed the experiments. YY, SJ and LF carried out the experiments. YY, SJ, LF, JZ, ZZ, ML, SZ, JO, WL, WG, XW, JW, DS and QZ analyzed the data. YY, DS, and QZ wrote the paper and finalized the manuscript. All authors read and approved the final manuscript.

\section{Compliance with Ethical Standards}

Conflict of interest The authors declare that they have no conflict of interest.

Animal and Human Rights Statement The whole study was approved by the Southern Medical University Animal Ethics Committee. All institutional and national guidelines for the care and use of laboratory animals were followed.

\section{References}

Alkhalaf MA, Guiver M, Cooper RJ (2015) Genome stability of adenovirus types 3 and 7 during a simultaneous outbreak in Greater Manchester, UK. J Med Virol 87:117-124

Baden LR, Walsh SR, Seaman MS, Johnson JA, Tucker RP, Kleinjan JA, Gothing JA, Engelson BA, Carey BR, Oza A, Bajimaya S, Peter L, Bleckwehl C, Abbink P, Pau MG, Weijtens M, Kunchai M, Swann EM, Wolff M, Dolin R, Barouch DH (2014) First-inhuman evaluation of a hexon chimeric adenovirus vector expressing HIV-1 Env (IPCAVD 002). J Infect Dis 210:1052-1061

Chang SY, Lee CN, Lin PH, Huang HH, Chang LY, Ko W, Chang SF, Lee PI, Huang LM, Kao CL (2008) A community-derived outbreak of adenovirus type 3 in children in Taiwan between 2004 and 2005. J Med Virol 80:102-112

Chen Y, Liu F, Wang C, Zhao M, Deng L, Zhong J, Zhang Y, Ye J, Jing S, Cheng Z, Guan Y, Ma Y, Sun Y, Zhu B, Zhang Q (2016) Molecular identification and epidemiological features of human adenoviruses associated with acute respiratory infections in hospitalized children in Southern China, 2012-2013. PLoS ONE 11:e0155412

Cheng Z, Yan Y, Jing S, Li WG, Chen WW, Zhang J, Li M, Zhao S, Cao N, Ou J, Zhao S, Wu X, Cao B, Zhang Q (2018) Comparative genomic analysis of re-emergent human adenovirus type 55 pathogens associated with adult severe communityacquired pneumonia reveals conserved genomes and capsid proteins. Front Microbiol 9:1180

Coleman KK, Wong CC, Jayakumar J, Nguyen TT, Wong AWL, Yadana S, Thoon KC, Chan KP, Low JG, Kalimuddin S, Dehghan S, Kang J, Shamsaddini A, Seto D, Su YCF, Gray GC (2019) Adenoviral infections in Singapore: should new antiviral therapies and vaccines be adopted? J Infect Dis. 221:566-577

Crawford-Miksza L, Schnurr DP (1996) Analysis of 15 adenovirus hexon proteins reveals the location and structure of seven hypervariable regions containing serotype-specific residues. J Virol 70:1836-1844

Duffy MR, Alonso-Padilla J, John L, Chandra N, Khan S, Ballmann MZ, Lipiec A, Heemskerk E, Custers J, Arnberg N, Havenga M, Baker AH, Lemckert A (2017) Generation and characterization of a novel candidate gene therapy and vaccination vector based on human species D adenovirus type 56. J Gen Virol. 99:135-147

Echavarría M (2009) Adenoviruses. In: Zuckerman AJ, Banatvala JE, Schoub BD, Griffiths PD, Mortime P (eds) Principles and practice of clinical virology, 6th edn. Wiley, Chichester, pp 463-488

Feng Y, Sun X, Ye X, Feng Y, Wang J, Zheng X, Liu X, Yi C, Hao M, Wang Q, Li F, Xu W, Li L, Li C, Zhou R, Chen L, Feng L (2018) Hexon and fiber of adenovirus type 14 and 55 are major targets of neutralizing antibody but only fiber-specific antibody contributes to cross-neutralizing activity. Virology 518:272-283

Fujimoto T, Hamamoto I, Taniguchi K, Chikahira M, Okabe N (2008) Molecular epidemiology of adenovirus type 3 detected from 
1994 to 2006 in Hyogo Prefecture, Japan. Jpn J Infect Dis 61:143-145

Gahery-Segard H, Farace F, Godfrin D, Gaston J, Lengagne R, Tursz T, Boulanger P, Guillet JG (1998) Immune response to recombinant capsid proteins of adenovirus in humans: antifiber and anti-penton base antibodies have a synergistic effect on neutralizing activity. J Virol 72:2388-2397

Ginn SL, Amaya AK, Alexander IE, Edelstein M, Abedi MR (2018) Gene therapy clinical trials worldwide to 2017: an update. J Gene Med 20:e3015

Gu L, Krendelchtchikova V, Krendelchtchikov A, Oster RA, Fujihashi K, Matthews QL (2014) A recombinant adenovirusbased vector elicits a specific humoral immune response against the V3 loop of HIV-1 gp120 in mice through the "Antigen Capsid-Incorporation" strategy. Virol J 11:112

Han G, Niu H, Zhao S, Zhu B, Wang C, Liu Y, Zhang M, Yang S, Liu F, Wan C, Zhang Q (2013) Identification and typing of respiratory adenoviruses in Guangzhou, Southern China using a rapid and simple method. Virol Sin 28:103-108

Harley D, Harrower B, Lyon M, Dick A (2001) A primary school outbreak of pharyngoconjunctival fever caused by adenovirus type 3. Commun Dis Intell 25:9-12

Holterman L, Vogels R, van der Vlugt R, Sieuwerts M, Grimbergen J, Kaspers J, Geelen E, van der Helm E, Lemckert A, Gillissen G, Verhaagh S, Custers J, Zuijdgeest D, Berkhout B, Bakker M, Quax P, Goudsmit J, Havenga M (2004) Novel replicationincompetent vector derived from adenovirus type 11 (Ad11) for vaccination and gene therapy: low seroprevalence and non-crossreactivity with Ad5. J Virol 78:13207-13215

Jing S, Zhang J, Cao M, Liu M, Yan Y, Zhao S, Cao N, Ou J, Ma K, Cai X, Wu J, Mei Y-F, Zhang Q (2019) Household transmission of human adenovirus type 55 in case of fatal acute respiratory disease. Emerg Infect Dis 25:1756-1758

Lebeck MG, McCarthy TA, Capuano AW, Schnurr DP, Landry ML, Setterquist SF, Heil GL, Kilic S, Gray GC (2009) Emergent US adenovirus 3 strains associated with an epidemic and serious disease. J Clin Virol 46:331-336

Lee J, Choi EH, Lee HJ (2010) Comprehensive serotyping and epidemiology of human adenovirus isolated from the respiratory tract of Korean children over 17 consecutive years (1991-2007). J Med Virol 82:624-631

Li L, Woo YY, de Bruyne JA, Nathan AM, Kee SY, Chan YF, Chiam CW, Eg KP, Thavagnanam S, Sam IC (2018) Epidemiology, clinical presentation and respiratory sequelae of adenovirus pneumonia in children in Kuala Lumpur, Malaysia. PLoS One 13:e0205795

Lin GL, Lu CY, Chen JM, Lee PI, Ho SY, Weng KC, Huang LM, Chang LY (2019) Molecular epidemiology and clinical features of adenovirus infection in Taiwanese children, 2014. J Microbiol Immunol Infect 52:215-224

Lion T (2014) Adenovirus infections in immunocompetent and immunocompromised patients. Clin Microbiol Rev 27:441-462

Liu TC, Kirn D (2008) Gene therapy progress and prospects cancer: oncolytic viruses. Gene Ther 15:877-884

Lu L, Zhong H, Su L, Cao L, Xu M, Dong N, Xu J (2017) Detection and molecular characterization of human adenovirus infections among hospitalized children with acute diarrhea in Shanghai, China, 2006-2011. Can J Infect Dis Med Microbiol 2017:9304830

Milligan ID, Gibani MM, Sewell R, Clutterbuck EA, Campbell D, Plested E, Nuthall E, Voysey M, Silva-Reyes L, McElrath MJ, De Rosa SC, Frahm N, Cohen KW, Shukarev G, Orzabal N, van Duijnhoven W, Truyers C, Bachmayer N, Splinter D, Samy N, Pau MG, Schuitemaker H, Luhn K, Callendret B, Van Hoof J, Douoguih M, Ewer K, Angus B, Pollard AJ, Snape MD (2016) Safety and immunogenicity of novel adenovirus type 26- and modified vaccinia ankara-vectored Ebola vaccines: a randomized clinical trial. JAMA 315:1610-1623

Pan H, Yan Y, Zhang J, Zhao S, Feng L, Ou J, Cao N, Li M, Zhao W, Wan C, Ismail AM, Rajaiya J, Chodosh J, Zhang Q (2018) Rapid construction of a replication-competent infectious clone of human adenovirus type 14 by gibson assembly. Viruses 10:568

Pena L, Moraes MP, Koster M, Burrage T, Pacheco JM, Segundo FD, Grubman MJ (2008) Delivery of a foot-and-mouth disease virus empty capsid subunit antigen with nonstructural protein $2 \mathrm{~B}$ improves protection of swine. Vaccine 26:5689-5699

Pereira SA, Florencio CM, Marinheiro JC, Harsi CM, Moura FE (2016) Adenoviruses and acute respiratory infections in children living in an equatorial area of Brazil. Epidemiol Infect 144:355-362

Peters W, Brandl JR, Lindbloom JD, Martinez CJ, Scallan CD, Trager GR, Tingley DW, Kabongo ML, Tucker SN (2013) Oral administration of an adenovirus vector encoding both an avian influenza A hemagglutinin and a TLR3 ligand induces antigen specific granzyme $\mathrm{B}$ and IFN-gamma $\mathrm{T}$ cell responses in humans. Vaccine 31:1752-1758

Philipson L (1961) Adenovirus assay by the fluorescent cell-counting procedure. Virology 15:263-268

Pichla-Gollon SL, Drinker M, Zhou X, Xue F, Rux JJ, Gao GP, Wilson JM, Ertl HC, Burnett RM, Bergelson JM (2007) Structure-based identification of a major neutralizing site in an adenovirus hexon. J Virol 81:1680-1689

Radin JM, Hawksworth AW, Blair PJ, Faix DJ, Raman R, Russell KL, Gray GC (2014) Dramatic decline of respiratory illness among US military recruits after the renewed use of adenovirus vaccines. Clin Infect Dis 59:962-968

Räty JK, Pikkarainen JT, Wirth T, Ylä-Herttuala S (2008) Gene therapy: the first approved gene-based medicines, molecular mechanisms and clinical indications. Curr Mol Pharmacol $1: 13-23$

Russell KL, Hawksworth AW, Ryan MA, Strickler J, Irvine M, Hansen CJ, Gray GC, Gaydos JC (2006) Vaccine-preventable adenoviral respiratory illness in US military recruits, 1999-2004. Vaccine 24:2835-2842

Rux JJ, Burnett RM (2000) Type-specific epitope locations revealed by X-ray crystallographic study of adenovirus type 5 hexon. Mol Ther 1:18-30

Rux JJ, Kuser PR, Burnett RM (2003) Structural and phylogenetic analysis of adenovirus hexons by use of high-resolution X-ray crystallographic, molecular modeling, and sequence-based methods. J Virol 77:9553-9566

Scallan CD, Tingley DW, Lindbloom JD, Toomey JS, Tucker SN (2013) An adenovirus-based vaccine with a double-stranded RNA adjuvant protects mice and ferrets against $\mathrm{H} 5 \mathrm{~N} 1$ avian influenza in oral delivery models. Clin Vaccine Immunol 20:85-94

Schooley RT, Spritzler J, Wang H, Lederman MM, Havlir D, Kuritzkes DR, Pollard R, Battaglia C, Robertson M, Mehrotra D, Casimiro D, Cox K, Schock B (2010) AIDS clinical trials group 5197: a placebo-controlled trial of immunization of HIV-1infected persons with a replication-deficient adenovirus type 5 vaccine expressing the HIV-1 core protein. J Infect Dis 202:705-716

Seto D, Chodosh J, Brister JR, Jones MS, MotAR Community (2011) Using the whole-genome sequence to characterize and name human adenoviruses. J Virol 85:5701-5702

Shukarev G, Callendret B, Luhn K, Douoguih M, Consortium E (2017) A two-dose heterologous prime-boost vaccine regimen eliciting sustained immune responses to Ebola Zaire could support a preventive strategy for future outbreaks. Hum Vaccin Immunother 13:266-270 
Singh G, Zhou X, Lee JY, Yousuf MA, Ramke M, Ismail AM, Lee JS, Robinson CM, Seto D, Dyer DW, Jones MS, Rajaiya J, Chodosh J (2015) Recombination of the epsilon determinant and corneal tropism: human adenovirus species D types $15,29,56$, and 69. Virology 485:452-459

Smaill F, Jeyanathan M, Smieja M, Medina MF, Thanthrige-Don N, Zganiacz A, Yin C, Heriazon A, Damjanovic D, Puri L, Hamid J, Xie F, Foley R, Bramson J, Gauldie J, Xing Z (2013) A human type 5 adenovirus-based tuberculosis vaccine induces robust $\mathrm{T}$ cell responses in humans despite preexisting anti-adenovirus immunity. Sci Transl Med 5:205ra134

Stecher H, Carlson CA, Shayakhmetov DM, Lieber A (2003) Generation of adenovirus vectors devoid of all viral genes by recombination between inverted repeats. Methods Mol Med $76: 135-152$

Stone D, Ni S, Li Z-Y, Gaggar A, DiPaolo N, Feng Q, Sandig V, Lieber A (2005) Development and assessment of human adenovirus type 11 as a gene transfer vector. J Virol 79:5090-5104

Tian X, Liu M, Su X, Jiang Z, Ma Q, Liao X, Li X, Zhou Z, Li C, Zhou R (2015) Mapping the epitope of neutralizing monoclonal antibodies against human adenovirus type 3 . Virus Res 208:66-72

Toth K, Wold WSM (2010) Increasing the efficacy of oncolytic adenovirus vectors. Viruses 2:1844-1866

Tsou TP, Tan BF, Chang HY, Chen WC, Huang YP, Lai CY, Chao YN, Wei SH, Hung MN, Hsu LC, Lu CY, Shao PL, Mu JJ, Chang LY, Liu MT, Huang LM (2012) Community outbreak of adenovirus, Taiwan, 2011. Emerg Infect Dis 18:1825-1832

Wo Y, Lu Q-B, Huang D-D, Li X-K, Guo C-T, Wang H-Y, Zhang X-A, Liu W, Cao W-C (2014) Epidemical features of HAdV-3 and HAdV-7 in pediatric pneumonia in Chongqing, China. Adv Virol 160:633-638

Wold WS, Toth K (2013) Adenovirus vectors for gene therapy, vaccination and cancer gene therapy. Curr Gene Ther 13:421-433

Yeung R, Eshaghi A, Lombos E, Blair J, Mazzulli T, Burton L, Drews SJ (2009) Characterization of culture-positive adenovirus serotypes from respiratory specimens in Toronto, Ontario, Canada: September 2007-June 2008. Virol J 6:11
Yu Z, Zeng Z, Zhang J, Pan Y, Chen M, Guo Y, Yu N, Chodosh J, Fu N, Che X, Zhang Q (2016) Fatal community-acquired pneumonia in children caused by re-emergent human adenovirus $7 \mathrm{~d}$ associated with higher severity of illness and fatality rate. Sci Rep 6:37216

Zhang Q, Su X, Gong S, Zeng Q, Zhu B, Wu Z, Peng T, Zhang C, Zhou R (2006) Comparative genomic analysis of two strains of human adenovirus type 3 isolated from children with acute respiratory infection in southern China. J Gen Virol 87:1531-1541

Zhang Q, Su X, Seto D, Zheng B-J, Tian X, Sheng H, Li H, Wang Y, Zhou R (2009) Construction and characterization of a replication-competent human adenovirus type 3-based vector as a livevaccine candidate and a viral delivery vector. Vaccine 27:1145-1153

Zhang Q, Jing S, Cheng Z, Yu Z, Dehghan S, Shamsaddini A, Yan Y, Li M, Seto D (2017) Comparative genomic analysis of two emergent human adenovirus type 14 respiratory pathogen isolates in China reveals similar yet divergent genomes. Emerg Microbes Infect 6:e92

Zhang J, Kang J, Dehghan S, Sridhar S, Lau S, Ou J, Woo P, Zhang Q, Seto D (2019) A survey of recent adenoviral respiratory pathogens in Hong Kong reveals emergent and recombinant human adenovirus type 4 (HAdV-E4) circulating in civilian populations. Viruses 11:129

Zhang J, Sridhar S, Lau SKP, Liu J, Ou J, Yan Y, Zhao S, Lan W, Guan W, Wu J, Woo PCY, Seto D, Zhang Q (2020) Molecular typing of human respiratory adenoviruses with universal PCR and sequencing primers for three major capsid genes: penton base, hexon, and fiber. BioRxiv. https://doi.org/10.1101/2020.03. 17.995373

Zhao S, Wan C, Ke C, Seto J, Dehghan S, Zou L, Zhou J, Cheng Z, Jing S, Zeng Z, Zhang J, Wan X, Wu X, Zhao W, Zhu L, Seto D, Zhang Q (2014) Re-emergent human adenovirus genome type 7d caused an acute respiratory disease outbreak in Southern China after a twenty-one year absence. Sci Rep 4:7365

Zhao Y, Lu R, Shen J, Xie Z, Liu G, Tan W (2019) Comparison of viral and epidemiological profiles of hospitalized children with severe acute respiratory infection in Beijing and Shanghai, China. BMC Infect Dis 19:729 